\title{
L'évolution de l'adoption en France : entre filiation et parentalité
}

L'approche historique et anthropologique des usages de l'adoption que je me propose de brosser à grands traits montre que cette institution a changé de contenu. Sous l'effet des logiques propres à chacun des acteurs sociaux, les parents adoptifs, l'Etat, et plus récemment les adoptés et les parents de naissance, l'institution de filiation s'est transformée peu à peu en une institution de parentalité ${ }^{1}$. Si ces deux concepts se recouvrent en partie, ils sont néanmoins distincts et je tenterai de montrer en quoi la réduction du deuxième au premier, entraîne contradictions et difficultés nouvelles.

\section{Evolution historique de l'institution}

\section{- de la recherche d'un descendant héritier...}

Il faut tout d'abord insister sur l'absence remarquable de l'adoption comme institution juridique de filiation en Europe de l'Ouest depuis le haut Moyen Age. Elle témoigne de l'hostilité de cette société à l'introduction d'un étranger au sang au sein d'une lignée. Cependant, en l'absence de progéniture, des contrats passés devant notaire réalisent des adoptions de fait. Un ou des parents sans enfants adoptent un descendant destiné à perpétuer la lignée en héritant des biens et parfois du nom, et à les assister dans leur vieillesse. L'adoption n'apparaît dans le droit français qu'en 1804 avec le Code Civil. La référence explicite des juristes de l'époque est l'adoption antique, grecque et romaine, dont ils partagent l'esprit : l'institution ne devant pas concurrencer le mariage légitime, seules peuvent adopter les personnes sans enfant légitime, âgées de plus de 50 ans (âge auquel elles n'espèrent plus avoir de descendants naturels). Elles adoptent des enfants majeurs (25 ans) auxquels elles doivent avoir " dans leur minorité et pendant six ans au moins fourni des secours et donné des soins ininterrompus ». Elles leur transmettent leurs biens et leur nom, ce dernier devant figurer après celui du père naturel de l'adopté. En effet, les adoptés restent dans leur famille naturelle, ce qui signifie dans les faits qu'ils gardent leur droit à la succession de leurs parents et conservent leur nom d'origine. Tout au long du XIXe siècle, les adoptants (à peine une centaine de cas par an pour toute la France pendant presque tout le siècle) - sont dans leur majorité des possédants, propriétaires rentiers, commerçants, ou membres de professions libérales qui veulent transmettre leurs biens, dans la moitié des cas à leurs enfants naturels (reconnus ou non reconnus) qu'ils légitiment ainsi, et secondairement aux enfants de leur conjoint ${ }^{2}$. Notons un fait important : les adoptés ne sont pas des enfants abandonnés. Ils ont généralement vécu avec un parent de naissance ou avec les deux qui eux-mêmes connaissent et estiment leurs parents adoptifs, l'adoption se faisant souvent dans la parenté ou parmi ses proches. Un couple sans enfant peut demander à un frère ou une sœur de l'un ou l'autre conjoint, ou à un ami de lui «donner » un de ses enfants ${ }^{3}$ en adoption.

\section{-...à celle d'un fils ou d'une fille à chérir}

Emerge progressivement une autre finalité de l'adoption : il n'est plus seulement question pour les adoptants de rechercher un successeur légitime mais aussi un enfant à chérir. Tout au long du XIXè siècle, s'affirme l'expression d'un " désir d'enfant " et pas seulement d'un désir

\footnotetext{
${ }^{1}$ Pour une analyse juridique de cette évolution, voir C.Neirinck, « L’évolution de l'adoption », in A.Fine et C.Neirinck (dir.), Parents de sang, parents adoptifs. Approches juridique et anthropologique de l'adoption. France, Europe, Canada, USA. Paris, LGDJ, 2000, p. 343-361.

2 J.P.Gutton, L'adoption, Paris, Publisud, 1993.

${ }^{3}$ A.Fine, «Le don d'enfants dans l'ancienne France », in A.Fine (dir.), Adoptions. Ethnologie des parentés choisies, Paris, Maison des sciences de l'homme, 1998, p. 61-95.
} 
de descendance, tandis que la défiance à l'égard de l'intégration d'un sang étranger au sein de la famille tend à s'atténuer. En témoigne la littérature de cette époque, par exemple les contes de Maupassant (1882). Dans l'un d'eux, l'auteur met en scène une jeune femme aristocrate mariée et sans enfant, tombant en admiration devant un groupe d'enfants qui jouent devant leur pauvre chaumière. Elle en choisit un, puis obtient l'accord de ses parents pour l'élever et l'adopter à sa majorité. Ici l'adoption est présentée comme une façon pour une mère d'assouvir un désir d'enfant. La loi de 1923, votée après la Grande Guerre qui avait multiplié le nombre des orphelins, permet de combler ce désir en autorisant un couple, désormais plus jeune, - l'âge légal des adoptants ayant été abaissé de 50 à 40 ans-, à élever et adopter un enfant (et non plus un adulte). Le nombre des adoptions augmente de manière importante, et la part relative des couples parmi les adoptants augmente elle aussi régulièrement. Le contenu nouveau qu'exprime l'adoption des mineurs ne s'oppose pas à la fonction successorale de l'adoption. Ces deux finalités se recouvrent et sont difficilement dissociables.

\section{-Un enfant dont on connaît les parents}

La nouvelle de Maupassant mettant en scène la remise d'un enfant à ses parents adoptifs par ses parents naturels, correspond au moins sur ce point précis à une réalité sociologique à cette époque et même plus tardivement. Selon un sondage dans les archives des adoptions pratiquées dans deux départements méridionaux entre 1923 et $1960^{4}$, les adoptants étaient plutôt des personnes assez âgées, entre 40 et 70 ans, qui adoptaient des adultes qu'elles avaient généralement élevés depuis leur enfance : le plus souvent des enfants de conjoints (plus de 60\% des adoptions simples en région parisienne au début des années 60), des neveux ou des nièces (plus de 20\% des cas), parfois des enfants de voisins ou d'amis, issus d'une famille nombreuse. L'adoption était « inspirée par un esprit testamentaire » dans le sens où l'adoptant, sans descendant, refusant la dispersion de ses biens, préférait les transmettre à un seul enfant, comme c'est d'ailleurs la coutume dominante dans nombre de régions françaises, en particulier dans le Midi. Si la loi prévoyait que les enfants adoptés pouvaient hériter aussi de leurs propres parents, la coutume s'y opposait. Les bénéfices patrimoniaux de ce don d'enfant devaient profiter à tous les enfants, en particulier à ceux qui restaient avec leurs parents de sang. Les futurs adoptés arrivaient chez leurs futurs parents adoptifs entre 2 et 12 ans, l'âge moyen se situant autour de 6 ou 7 ans. Ils connaissaient donc parfaitement leurs parents et, lorsqu'ils étaient les neveux ou nièces de leurs parents adoptifs, ils s'adressaient à eux avec les termes de parenté appropriés (oncle ou tante) tandis qu'ils continuaient à appeler leurs propres parents papa, maman. Dans l'adoption du neveu ou de la nièce, des relations affectives préexistaient à l'établissement de la vocation successorale de l'adopté. C'est vrai aussi des enfants de conjoints adoptés : $2 / 3$ avaient perdu l'un de leurs parents au moment de l'adoption et avaient été probablement élevés une partie de leur enfance par leur mère avec son nouveau conjoint, 1/3 étaient des enfants de conjoints divorcés, et dans ce cas, leur parent avait dû consentir à leur adoption ${ }^{5}$. Souvent le parent adoptif était une femme qui, en élevant un enfant mineur, était poussée par le désir de nouer avec lui des relations affectives privilégiées. Les femmes étaient en effet nombreuses à élever seules et à adopter des enfants. Entre 1923 et 1960, sur 255 adoptions qui ont eu lieu dans le département du Tarn, 58\% ont été réalisées par des personnes seules dont les deux tiers sont des femmes, surtout des veuves, et en nombre moins important des célibataires. Les adoptés conservaient leur filiation d'origine, ils continuaient à porter le nom de leur père de naissance auquel ils ajoutaient celui

\footnotetext{
${ }^{4}$ Agnès Galibert, Formes et enjeux de l'adoption ancienne, à partir d'exemples tarnais (1923-fin des années 1960), Maîtrise d'histoire sous la direction d'A.Fine, Université de Toulouse-Le Mirail, 1999.

${ }^{5}$ Enquêtes menées dans la région parisienne au début des années 1960 par Marie-Pierre Marmier, Sociologie de l'adoption. Etude de sociologie juridique. Thèse pour le doctorat en droit, Paris, 1969. Voir p. 40 et ss.
} 
de leur père adoptif. Notons que l'adoption additive, inclusive, était une forme particulièrement adaptée à l'adoption tardive, ainsi qu'à l'adoption par des personnes seules.

\section{-Un enfant sans parents proposé par l'Etat : le modèle de substitution}

Pendant la même période, apparaît une nouvelle forme d'adoption, celle d'enfants petits, orphelins ou abandonnés, majoritairement adoptés par des couples stériles. Elle fonde sur le plan juridique une nouvelle finalité de l'adoption, donner une famille à un enfant qui en est dépourvu. Ces enfants sans parents font l'objet d'une législation spécifique, la légitimation adoptive, interdite aux personnes seules. En effet, en 1939, le code de la famille prévoit que seuls les couples mariés depuis plus de 10 ans sans enfants et dont l'un des deux a au moins 35 ans peuvent adopter des enfants de moins de cinq ans, abandonnés ou dont les parents sont inconnus ou décédés, en en faisant leurs enfants légitimes. La mention de la légitimation adoptive est faite en marge de l'acte de naissance de l'enfant qui a les mêmes droits et les mêmes obligations que s'il était né du mariage. L'adopté ne porte que le nom de son père adoptif ${ }^{6}$. Une coupure définitive avec la famille naturelle est instaurée, qui sera consacrée par une nouvelle forme d'adoption, l'adoption plénière en 1966.

Cette nouvelle forme d'adoption voit le jour grâce à l'intervention d'un nouvel acteur, l'Etat, représenté par les services administratifs de la protection de l'enfance. Notons en effet que c'est seulement à cette époque, l'entre-deux-guerres, que l'histoire de l'abandon et celle de l'adoption, longtemps parallèles, se sont croisées de manière durable. Auparavant en effet, non seulement les enfants adoptés n'étaient pas des enfants abandonnés, mais ces derniers n'étaient pas adoptés. De fait l'adoption n'était pas pensée comme une solution à la question de l'enfance assistée.

A partir de 1923, l'Etat prend l'initiative de placer en adoption les enfants orphelins et abandonnés. L'échange direct entre protagonistes étant exclu, pour être adopté, un enfant doit donc d'abord être abandonné, de sorte que si l'on admet la succession de plusieurs parents dans l'histoire d'un enfant, on refuse l'idée d'une possible coexistence, non seulement en droit, mais aussi dans la réalité de la vie de l'enfant. C'est ainsi que "l'effacement de la famille d'origine " a été recherché explicitement par le législateur dès 1923 et qu'il a été érigé en principe législatif en 1939 en France avec la légitimation adoptive, puis définitivement consacré en 1966 avec la loi sur l'adoption plénière. Ces textes qui consacrent l'usage de l'adoption comme forme substitutive de procréation pour les couples sans enfant, ainsi que la conjoncture nataliste de l'époque, expliquent la multiplication du nombre des adoptions à partir de 1943, ce type d'adoption ayant rapidement pris le pas sur l'adoption classique en ce qui concerne les petits enfants. Les légitimations adoptives représentent $35 \%$ de l'ensemble des adoptions en 1952, 43\% en 1966. Les enfants adoptés sont essentiellement des pupilles de l'Etat, orphelins ou abandonnés, généralement très petits, tandis que les adoptants sont des couples stériles, heureux de satisfaire leur besoin de paternité et surtout de maternité dans les conditions les plus proches de la procréation naturelle, c'est à dire, sans la présence ou la concurrence de parents de sang. En instituant l'adoption plénière, bien distinguée dans ses effets de l'ancienne adoption qui devient désormais l'adoption simple, la loi de 1966 consacre ce nouveau modèle. Votée pour mettre un terme définitif à la possible concurrence entre parents adoptifs et parents de sang qu'avait posée de manière cruciale et très médiatisée l'affaire Novack ${ }^{7}$, l'adoption plénière est exclusive en ce sens que tout lien légal entre l'adopté et sa famille d'origine est coupé. Les parents adoptifs deviennent les seuls et uniques parents de l'enfant adopté qui porte désormais uniquement leur nom, son état civil

\footnotetext{
${ }^{6}$ Pour la première fois, la loi de 1949 donne aux parents adoptifs la possibilité de changer les prénoms des adoptés.

${ }^{7}$ L'affaire Novack est un conflit autour d'un enfant que ses parents naturels veulent reprendre à ses parents adoptifs.
} 
modifié laisse penser qu'il est né de ses parents adoptifs. L'adoption simple des enfants passe au second plan, elle concerne essentiellement l'adoption de l'enfant de conjoint. En quelques années, l'adoption plénière devient la forme dominante d'adoption. Aujourd'hui plus de $90 \%$ des couples qui adoptent des enfants petits, orphelins ou abandonnés français et étrangers 8 usent de cette forme légale. L'adoption plénière concerne aussi les adoptants vivant seuls qui peuvent désormais être les seuls parents légaux d'un enfant, ce qui n'était pas le cas auparavant. Le parent seul ne se vit pas seulement comme un deuxième parent, partageant sa paternité ou sa maternité avec d'autres, mais comme le seul parent. Cette adoption n'a manifestement plus le même contenu que celle que nous avons décrite précédemment.

L'usage de l'institution du point de vue des parents, se combine à celui qu'en font les services de protection de l'enfance pour lui donner un contenu nouveau, ce qui ne va sans entraîner difficultés et contradictions, dans les faits et dans le droit.

\section{Une nouvelle définition de l'enfant et de la famille}

Je voudrais présenter ici l'analyse que l'anthropologue F-R.Ouellette ${ }^{9}$ propose à partir de l'observation des usages de l'adoption au Québec, car elle me semble valoir également pour éclairer la situation française, et plus généralement en Occident. Elle montre comment les logiques de fonctionnement des services de protection de l'enfance contribuent à forger une nouvelle définition de l'enfant et des parents. Pour mieux préserver l'intérêt de l'enfant dont la sécurité et le développement sont compromis parce qu'il a été abandonné ou parce qu'il ne reçoit pas de ses parents les soins adéquats, les services sociaux cherchent à lui procurer, grâce à l'adoption, une famille stable avec des parents évalués pour leur capacité parentale. L'enfant est donc défini avant tout comme une personne mineure à protéger et l'adoption comme un transfert des droits et responsabilités parentales à l'égard de l'enfant. La rupture de la filiation d'origine et son impact identitaire restent dans l'ombre ; elle apparaît comme un élément secondaire dans une optique de protection centrée sur les besoins immédiats de l'enfant. Comme le souligne F-R.Ouellette, la position généalogique de l'enfant n'est pas appelée à le définir, même si l'adoption consiste bel et bien à le déloger de cette position pour l'intégrer dans un autre réseau de parenté, ce qui lui confère une nouvelle identité. Dans cette optique, l'adoption peut être valorisée même pour des enfants d'un certain âge, y compris avec filiation établie, et même pour des enfants dont les parents refusent l'adoption.

Cette idée que l'enfant est un individu autonome, présuppose aussi que l'enfant devienne «disponible », ce qui est accompli par l'acte d'abandon. Or ces deux opérations, le consentement à l'adoption des parents, ou la déclaration judiciaire d'admissibilité à l'adoption, d'une part, l'ordonnance de placement en vue d'adoption d'autre part, sont effectuées le plus souvent par des équipes différentes. Ces pratiques, vraies pour le Québec, mais également pour la France, concourent à façonner l'image d'un « enfant sans attaches », aussi bien pour l'équipe qui cherche à le faire adopter que pour les parents adoptants dans la mesure où il n'est prévu aucun contact entre adoptants et parents d'origine.

L'enfant issu de l'accouchement sous X, une originalité française, est l'expression poussée à son terme de cette « organisation légale et administrative qui atomise complètement l'enfant, le fait délibérément venir au monde en dehors de tout lien de filiation », pour en faire l'enfant de «personne ». Dans l'adoption internationale, la distance permet davantage encore de penser que l'enfant est sans attache et non pas le fils d'un tel et une telle. Plusieurs images,

\footnotetext{
${ }^{8}$ En 1992, près de 93\% des adoptions plénières concernant des enfants étrangers ou pupilles de l'Etat étaient réalisées par des couples. cf Infostat Justice, septembre 1996, n 46.

${ }^{9}$ Françoise-Romaine Ouellette, «L'adoption face aux redéfinitions de la famille et de l'institution généalogique », A.Fine et C.Neirinck (dir.), Parents de sang, parents adoptifs, op.cit, p 325-341.
} 
celle de l'enfant trouvé, de l'enfant de la rue, de la planète, du Monde, de l'Orient, concourent à se représenter, en Occident, l'enfant adopté comme un enfant sans parents. Parfois des rencontres ont lieu entre adoptants et parents de sang, mais elles sont sans suite parce que les adoptants ne le souhaitent généralement pas.

La logique fonctionnelle des services sociaux concourt aussi à façonner une définition particulière des parents. Le parent recherché est un adulte capable de subvenir aux besoins matériels et affectifs de l'enfant. Or l'adoption confère aux adoptants une nouvelle position généalogique de père ou de mère, situant ainsi l'enfant à une place spécifique dans l'ordre des générations et dans l'univers de la parenté. Cet aspect est perçu comme secondaire. C'est plutôt l'accès d'un homme ou d'une femme à la parentalité en tant que rôle social de protection et forme valorisée d'épanouissement personnel qui s'avère constituer l'enjeu central de l'adoption pour les adoptants comme pour les services sociaux. F.R.Ouellette en veut pour preuve que les termes «parents » et «famille» sont interchangeables. On dira qu'on recherche une famille d'adoption ou encore des parents adoptifs pour tel ou tel enfant. Les termes «parents » sont utilisés plutôt que ceux de père et mère et l'on tend ainsi à dissoudre la problématique de la paternité et de la maternité dans l'idée générale de «parentalité ». Selon F-R. Ouellette, cette interchangeabilité des termes reflète une tendance à l'effacement de la dimension structurelle de la filiation dont la portée principale est justement de différencier la position de chacun dans l'ordre des générations. Cette définition de la famille fait que l'articulation de l'enfant à l'organisation généalogique de sa parenté d'origine paraît secondaire. D'ailleurs les parents qui n'ont pas la garde de leurs enfants, sont vus comme extérieurs à la famille de sorte que leur statut s'en trouve fragilisé sinon compromis. Un parent défaillant n'est plus considéré comme un parent. Dans cette logique, les services sociaux peuvent même favoriser une politique d'abandon, lorsque les parents sont jugés insatisfaisants.

Autrement dit, la famille est perçue comme un espace éducatif et le lien de filiation tend à se réduire au lien parent/enfant, et semble n'être plus fondé que sur le «projet parental». Les notions de filiation et de parentalité sont confondues alors que la «parentalité », néologisme apparu relativement récemment dans les sciences sociales et humaines, ne recouvre que le champ des relations parents/enfants, une partie seulement de celui de la parenté qui est beaucoup plus étendu, en profondeur, en collatéralité, du côté paternel et du côté maternel. Le terme parentalité n'existe pas en droit qui ne connaît que les termes sexués de père et de mère, relatifs à la filiation, soit à l'inscription d'un individu dans une organisation généalogique, un système de parenté. Selon F-R. Ouellette, «le lien parent/enfant tend à occuper tout l'espace de la filiation » et l'on assiste à une disqualification de l'organisation généalogique qui nous sert culturellement d'ancrage pour symboliser la différence des sexes, des âges et des générations. Elle se réalise sur la base d'une dissociation, d'une désimbrication conceptuelle entre famille et organisation généalogique de la parenté ${ }^{10}$.

Quelles sont les difficultés ou contradictions induites par le nouveau contenu donné à l'ancienne institution? Elles sont nombreuses : par exemple la possibilité légale faite aux célibataires d'adopter face à l'interdiction opposée aux couples concubins ou homosexuels ${ }^{11}$, la question de l'irrévocabilité de l'adoption plénière dans les cas d'échec de l'adoption. Je n'en évoquerai qu'une, qui me paraît plus proche des enjeux de ce colloque, les limites de l'adoption plénière dans l'adoption tardive.

\footnotetext{
${ }^{10}$ F.R.Ouellette, « Les usages contemporains de l'adoption », in Adoptions. Ethnologie des parentés choisies, op.cit, p 153-176.

${ }^{11}$ Sur ce point voir Agnès Fine, «Unifiliation ou double filiation dans l'adoption française », Anthropologie et sociétés, 2000, 24/3, p. 21-38.
} 


\section{Les limites de l'adoption plénière dans l'adoption tardive.}

Le modèle de substitution, fondé pendant longtemps sur le secret de l'adoption elle-même et le secret sur l'identité des parents d'origine de l'enfant, dont nous avons esquissé l'histoire, s'est fragilisé avec l'adoption internationale qui rend impossible le secret sur l'adoption. Il est actuellement remis en question dans la plupart des pays occidentaux et l'on peut penser que la France n'échappera pas à cette évolution. Depuis environ trente ans aux Etats-Unis, à la suite des actions organisées par des associations d'adoptés d'un côté ${ }^{12}$ et des associations de mères ayant abandonné leur enfant de l'autre, les premiers pour des raisons identitaires, les deuxièmes pour des raisons à la fois personnelles et politiques, s'est développé un mouvement pour les « retrouvailles » entre enfants et parents de sang, très médiatisées. Ce mouvement, relayé en Europe par le biais d'internet, a abouti à une transformation profonde du cadre juridique et pratique des adoptions dans ce pays, connue sous le nom d'open adoption (adoption «ouverte» par opposition à l'adoption «fermée» traditionnelle). Elle concerne essentiellement l'adoption interne et non pas l'adoption internationale qui reste statistiquement la plus fréquente. L'ouverture consiste à favoriser l'interconnaissance entre les géniteurs et les parents adoptifs, sous les formes les plus variées, depuis la simple connaissance de leur identité respective (sans rencontre) à la fréquentation régulière (avec parfois droit de visite reconnu pour les parents de sang), le contrat étant négocié entre les partenaires. D'une part les agences favorisent le choix par les mères de naissance des futurs parents adoptifs de l'enfant, d'autre part elles mettent en place des sortes d'ententes morales entre les partenaires sur les suites que les uns et les autres souhaitent donner à leur mise en contact. Les débats son très vifs entre juristes sur le caractère exécutoire ou non de ces ententes. Depuis le milieu des années 80, on est passé de simples rendez-vous annuels avec la mère de naissance, à un système d'interactions permanent entre elle et la famille adoptive, les deux parties étant réellement impliquées dans l'éducation de l'enfant. Cette forme d'adoption a connu un succès grandissant et elle est actuellement la norme aux USA ${ }^{13}$. Elle s'étend au Canada, tandis qu'en Grande-Bretagne et en Allemagne, les services sociaux la mettent en pratique alors que la loi n'a pas encore changé. Son essor s'explique aussi par la pénurie d'enfants petits adoptables qui concerne la majorité des pays occidentaux : elle présente en effet un caractère attractif pour les mères d'origine dans la mesure où elle tend à dédramatiser l'abandon, présenté comme un don altruiste d'un enfant qui gardera des relations avec elles. Elle est également un instrument politique de lutte contre l'avortement.

Il n'existe qu'une forme juridique d'adoption dans tous les pays cités, l'adoption plénière qui partout organise la rupture juridique de la première filiation de l'enfant. Les parents adoptifs sont donc bien les seuls parents légaux mais ici, la rupture juridique de la filiation n'entraîne pas ipso facto la disparition de contacts avec la parenté d'origine. Cette nouvelle pratique va de pair avec des transformations concernant également l'adoption de l'enfant du conjoint. En effet dans ce cas, la rupture totale des liens d'un enfant avec sa parenté d'origine même si elle est souhaitée par le conjoint séparé qui se remarie et veut normaliser sa nouvelle famille, peut être contraire à l'intérêt de l'enfant. Ce constat a conduit à alléger l'application de la loi, et dans certains Etats, des liens légaux peuvent désormais perdurer entre l'enfant adopté et sa famille naturelle, à travers la succession patrimoniale d'une part, le droit de visite d'autre part. Les contacts post-adoption de l'enfant avec la parenté dont il est séparé (grands-parents paternels par exemple) et même le père, font l'objet

\footnotetext{
${ }^{12}$ Adoption Rights Movement.

${ }^{13}$ Sur l'adoption ouverte, voir les contributions de E.Wayne Carp, de Joan Heifetz Hollinger pour les EtatsUnis, de Dominique Goubau pour le Canada, d'Eva Wenner pour l'Allemagne et Laurence Bacqué pour la Grande Bretagne in A.Fine et C.Neirinck (dir.), Parents de sang, parents adoptifs, op.cit.
} 
de grandes controverses. Des tribunaux reconnaissent des droits de visite au père d'origine séparé lorsqu'ils servent l'intérêt de l'enfant. L'adoption simple française paraît de ce point de vue bien mieux adaptée à ce type d'adoption.

L'élimination de la première filiation de l'enfant, apparaît comme encore plus problématique dans l'adoption tardive, interne et externe. Dans l'adoption interne, D.Goubau, juriste spécialiste de l'adoption au Canada, note «que le paradigme de l'adoption comme projet familial d'un couple, qui domine encore et toujours les normes légales en la matière dans son pays, cède graduellement le pas à l'adoption conçue comme un projet conjoint où se rencontrent deux familles autour d'un enfant dont l'une et l'autre recherchent le meilleur intérêt. La question du droit aux origines (possibilité légale de renouer avec ses parents naturels dès l'âge de 14 ans au Québec depuis 1994) oblige à reconsidérer en même temps celle de l'opportunité d'une rupture obligatoire des liens entre l'enfant et sa famille naturelle ${ }^{14}$. Il poursuit : «Dans le modèle traditionnel de l'adoption, la conception rigide de l'intérêt de l'enfant oblige à mettre en balance l'intérêt de l'enfant à être adopté et celui de maintenir des contacts avec un parent d'origine, l'un excluant l'autre. Pourtant il ne fait aucun doute que dans certains cas, il est dans l'intérêt de l'enfant d'être adopté tout en gardant certains contacts significatifs pour lui. On peut songer ici à la situation de l'enfant en famille d'accueil dont le parent naturel ne pourra jamais prendre soin mais qui a noué avec ce parent ou avec un autre membre de sa famille d'origine des liens que rien ne justifie de couper. L'adoption par la famille d'accueil peut en même temps représenter le meilleur projet de vie pour cet enfant. Dans tous les cas où le dilemme est réel, l'adoption ouverte apparaît alors comme la solution la plus respectueuse de l'intérêt de l'enfant, seul critère décisionnel acceptable. ${ }^{15}$

Cette analyse rejoint celle que viennent de publier trois chercheuses sur le fonctionnement d'une structure originale propre au Québec, «la banque mixte». Voici comment sont définis ses objectifs, qui ne concernent que l'adoption interne : «Elle vise à offrir un projet de vie stable à des enfants placés qui ont été victimes de négligence, d'abus ou de rejet et pour lesquels un retour auprès des parents d'origine est considéré comme improbable. Le programme s'adresse à des candidats à l'adoption qui acceptent d'une part, de jouer d'abord le rôle de famille d'accueil auprès de l'enfant avant qu'il ne devienne légalement adoptable; d'autre part, de prendre le risque que cet enfant retourne chez ses parents d'origine ou ne soit jamais légalement adopté. Il cherche des parents aptes à accueillir des enfants fréquemment caractérisés par des besoins spéciaux ${ }^{16}$.

Après avoir fait plusieurs études de cas approfondies, les auteures soulignent la nouveauté et l'intérêt du programme, mais elles concluent que "faute d'offrir d'autre issue légale que la rupture des liens avec la famille d'origine, les programmes de ce type reproduisent et renforcent la norme d'exclusivité qui interdit de penser que la stabilité familiale d'un enfant puisse se concilier avec la coexistence de plusieurs références parentales. Certaines situations de placement en « banque mixte » semblent pourtant tirer leur équilibre de cette coexistence. Leur fragilité tient au fait qu'aucun cadre légal ou d'intervention ne permet actuellement de reconnaître en même temps les liens d'origine et les liens d'adoption. Ce constat milite pour une réflexion approfondie sur les avantages possibles de l'adoption simple et de l'adoption ouverte. Les postulants à l'adoption y seraient probablement réticents au départ, mais l'expérience démontre qu'ils parviennent à ajuster

\footnotetext{
${ }^{14}$ Dominique Goubau, «Open adoption au Canada », in A.Fine et C.Neirinck, Parents de sang, parents adoptifs, op.cit, p. 63-85.

${ }^{15}$ Dominique Goubau, «Open adoption au Canada », op.cit, p. 85.

${ }^{16}$ Françoise-Romaine Ouellette et Caroline Méthot, Julie Paquette, « L'adoption, projet parental et projet de vie pour l'enfant. L'exemple de la « banque mixte » au Québec », Informations sociales, 107, 2003, p. 66-75.
} 
leurs attentes aux conditions concrètes d'accès aux enfants adoptables. Elle démontre aussi que leur désir d'exclusivité cède habituellement le pas au souci d'investir l'enfant qui a besoin d'eux ».

L'intérêt que nos collègues étrangers spécialistes de l'adoption portent à la forme légale de l'adoption simple française, dont j'ai esquissé l'histoire de son usage et les raisons de son délaissement relatif, nous invite à la réévaluer, et à éventuellement lui donner une place plus importante, en particulier dans les adoptions tardives. Il s'agirait alors d'admettre dans le domaine de l'adoption comme dans d'autres configurations familiales (par exemple dans les recompositions après divorce), qu'un enfant peut avoir besoin pour se construire de plusieurs figures parentales, à condition que le statut des unes et des autres soit clairement institué sur le plan juridique.

\title{
Résumé :
}

Cet article trace à grands traits l'évolution du droit et des usages de l'adoption depuis le début du XIXè siècle en France et montre comment on est passé d'une institution de filiation à une institution de parentalité, les parents adoptifs se substituant aux parents de sang défaillants. Ce modèle de substitution consacré par la forme juridique de l'adoption plénière et le délaissement de l'adoption simple est aujourd'hui remis en question dans plusieurs pays occidentaux avec l'adoption ouverte (open adoption). Ses limites sont particulièrement évidentes dans l'adoption tardive

\begin{abstract}
In this article, we describe the evolution of laws and practices in adoption, from the beginning of the XIX century in France : how the search of descent has evolved into search of parenthood, as adoptive parents took the place of genuine parents. This form of substitution, as we find it in legal exclusive adoption, is challenged nowadays in some occidental countries by open adoption. Its limits are particulary visible when adoption of older children is concerned.
\end{abstract}

\section{Mots clefs :}

Adoption, France, XIXè siècle, XXè siècle, law, pratices, descent, parenthood, open adoption, adoption of older children

\section{Bibliographie}

Adoption, The Future of Children, 1993, vol 3, n 1.

Corbier Mireille (dir), Adoption and Fosterage, Paris, De Boccard, 1999.

Gager Kristine, Blood Ties and Fictive Ties : Adoption and Family Life in Early Modern France, Princeton, Princeton University Press, 1996.

Lallemand Suzanne, La circulation des enfants en société traditionnelle. Prêt, don, échange, Paris, L'Harmattan, 1993.

Lammerant Isabelle, L'adoption et les droits de l'homme en droit comparé, Bruxelles/Paris, Bruylant/LGDJ, 2001.

Modell Judith, Kinship with Strangers : Adoption and Interpretations of Kinship in American Culture. Berkeley, University of California Press, 1994.

Ouellette Françoise-Romaine, L'adoption. Les acteurs et les enjeux autour de l'enfant. Sainte Foy, Les Presses de l'université de Laval, 1996.

Ouellette Françoise-Romaine et Frigault, L.R, Les adoptions internationales au Québec, Montréal, INRS, Culture et Société, 1996.

Ouellette Françoise-Romaine et Méthot Catherine, L'adoption tardive internationale. L'intégration familiale de l'enfant du point de vue des parents et des grands-parents, Montréal INRS-Culture et Société, 2000. 